\title{
A NEW LOCAL PROPERTY OF EMBEDDINGS
}

\author{
BY O. G. HARROLD ${ }^{1}$
}

Communicated June 30, 1965

It is known that the possible embeddings of a topological $n-1$ manifold $M^{n-1}$ in the euclidean space $E^{n}$ differ in the cases $n=3$ and $n>3$ in a curious way. A topological $n-1$ sphere can fail to be locally flat at an arbitrary finite number of points if $n=3$. For $n>3$ this cannot happen at a set consisting of a single point [2]. It is unresolved if an $S^{n-1}$ in $E^{n}$ can fail to be locally flat at a pair of points. In this note we introduce a new notion, described in detail below, called a locally weakly flat embedding and show that if a manifold $M^{n-1}$ in $E^{n}$ is locally flat at each point except possibly at the points of a finite set $Y$ and if $M^{n-1}$ is locally weakly flat at each point of $Y$, then $M^{n-1}$ is in fact locally flat at every point. In the concluding paragraph an unsolved problem is posed.

Let $p \in M^{k} \subset E^{n}$, or more generally $M^{k} \subset M^{n}$. Suppose $\epsilon>0$. Let $B_{a}^{n}$ be a ball of diameter less than $\epsilon$ whose interior contains $p$. For $0<t \leqq \epsilon$ let $B_{t}$ denote a ball whose interior contains $p$ and is concentric to $B_{e}^{n}$, i.e., regard $B_{t}$ as a topological product $S^{n-1} \times[0, t]$ with $S^{n-1} \times[0]$ identified with $p$. For all $t$ such that $\epsilon-t$ is sufficiently small we hypothesize that $\dot{B}_{t} \cap M$ is a $k-1$ sphere such that the pairs

$$
\left(E^{n}, \dot{B}_{t}^{n} \cap M^{k} \times I^{n-k+1}\right) \approx\left(E^{n}, S^{k-1} \times I^{n-k+1}\right)
$$

are homeomorphic. If for a sequence of positive numbers $\epsilon_{1}, \epsilon_{2}, \cdots$ converging to zero, this condition holds, we describe the embedding by saying $M^{k}$ is locally weakly flat at $p$. If this holds for all $p \in M^{k}$, $M^{k}$ is locally weakly flat in $M^{n}$, denoted by LWF.

A comparison with other local properties of embeddings [3] shows that $\mathrm{LF}=\mathrm{LU} \Rightarrow \mathrm{LWF} \Rightarrow \mathrm{LSPU} \Rightarrow \mathrm{LPU}$.

For $n=3, k=2$ these implications may be reversed [4]. There are examples, for $n=3$, that show that at a single point, local peripheral unknottedness, or local weakly flatness does not imply local flatness [5].

For $n=3, k=1, \mathrm{LU}$ and LPU are entirely independent. In this paper attention is restricted to $k=n-1$.

Theorem. Let $M^{n-1} \subset E^{n}$ be a closed $n-1$ manifold that is locally

1 Supported in part by NSF-Grant GP 4006. 
flat at each point except possibly at the points of a finite set $Y$. Suppose that $M^{n-1}$ is LWF at each point. Then $M^{n-1}$ is locally flat at each point.

The proof rests on an adaptation of a theorem of M. Brown's to what I refer to as the "Turning Lemma" for annuli. The same idea can be used to establish a "Union Lemma" for $n-1$ disks in $E^{n}$.

Notations. In order to ease our descriptions we define once and for all the meaning of

(1) nice $k$-disk in $S^{k}$, denoted by $D^{k}$;

(2) nice $k$-disk in $E^{k+1}$, denoted by $D^{k}$;

(3) nice $k$-annulus in $S^{k}$, denoted by $A^{k}$;

(4) nice $k$-annulus in $E^{k+1}$, denoted by $A^{k}$.

By (1) we mean the boundary $\partial D^{k}$ of $D^{k}$ has a shell neighborhood in $S^{k}$. By (2) we mean that $D^{k}$ is the image of an equatorial plane section under some homeomorphism of a standard $k+1$ ball into $E^{k+1}$. By (3) we mean each boundary component of $\partial A^{k}$, the boundary of $A^{k}$, has a shell neighborhood in $S^{k}$. By (4) we mean $A^{k}$ is the image of an equatorial plane section under some heomormophism of a standard $I^{2} \times S^{k-1}$ into $E^{k+1}$.

Some recent results needed for the proof. 1. Let $h$ be a homeomorphic embedding of $S^{n} \times[-1,1]$ into $S^{n+1}$, where $[a, b]$ denotes the closed real number interval $a \leqq t \leqq b$. Then the closure of either complementary domain of $h\left(S^{n} \times[0]\right)$ is an $(n+1)$-cell (Theorem 5 of $A$ proof of the generalized Schoenflies theorem, M. Brown).

2. Let $B$ be a subset of a metric space $X$. Suppose $B=U_{1} \cup U_{2}$, where $U_{1}, U_{2}$ are open in $B$ and $U_{1} \cap U_{2} \neq \square$. If both $U_{1}, U_{2}$ are collared in $X$, then $B$ is collared in $X$. If $B$ is an orientable bounded manifold of $\operatorname{dim} n$ in $E^{n+1}$, and $B$ is collared on each "side," $B$ is bicollared at each point of $B \backslash \partial B$. (Lemma 4 of Locally flat embeddings of topological manifolds, M. Brown [1]).

3. Let $D_{1}$ and $D_{2}$ be topological $n$-disks in $E^{n+1}$. Suppose each of $D_{1}$ and $D_{2}$ is nice (see above under Notations). Let $D_{1} \cap D_{2}=\partial D_{1} \cap \partial D_{2}$ $=S^{n-1}$. Suppose $S^{n-1}$ lies in the interior of a nice annulus $A$ that is a subset of $D_{1} \cup D_{2} .{ }^{2}$ Then $S=D_{1} \cup D_{2}$ is nice.

$3^{\prime}$. Let $\tilde{D}$ and $\tilde{A}$ be respectively a nice $n$-disk, a nice $n$-annulus in $E^{n+1}$. Suppose $\tilde{D} \cup \tilde{A}$ is a disk. Further $\partial \tilde{D}$ lies in Int $\tilde{A}$. Then $\tilde{D} \cup \tilde{A}$ is a nice disk in $E^{n+1}$. The proofs of 3 and $3^{\prime}$ are so similar to that of $3^{\prime \prime}$ we omit them.

2 The symbol "int" occurs in two senses. The meaning will be clear since in one case it means the bounded component of the complement of a set and in the other case it refers to the points not on the combinatorial boundary of some manifold with boundary. 
$3^{\prime \prime}$. The Turning Lemma. Let $F$ be a homeomorphism, $F: S^{n-1} \times I^{2}$ $\rightarrow E^{n+1}$. Let $I_{1}$ and $J_{1}$ be intervals lying in the interior of $I^{2}$ such that $I_{1} \cap J_{1}=\{0\}$, an endpoint of each of them. Suppose

(i) $F\left|S^{n-1} \times I_{1}=A_{1}, F\right| S^{n-1} \times J_{1}=A_{2}$, and

(ii) $F \mid S^{n-1} \times\{0\}=S^{n-1}$.

Then $A_{1} \cup A_{2}$ is nice in $E^{n+1}$.

To put it another way, whenever two $n$-annuli $A_{1}$ and $A_{2}$ are nice in $E^{n+1}$ and their common part is a component $S_{12}$ of the boundary of each of them, and if $F$ satisfies the consistency conditions (i) and (ii) above, then $A_{1} \cup A_{2}$ is nice.

Proof. Let $g$ be a homeomorphism of $I^{2}$ on $I^{2}$ so that $I_{1} \cup J_{1}$ is carried onto $I_{1} \cup J_{1}$ carrying $\{0\}$ into an inner point of $J_{1}$, leaving the other endpoints fixed, ${ }^{3}$ and also leaving the points of $S^{\prime}=\partial I^{2}$ fixed. Then

$$
G(x, y)=F(x, g(y))
$$

defines a homeomorphism of $S^{n-1} \times I^{2}$ onto $F\left(S^{n-1} \times I^{2}\right)$ and $A_{1}$ onto $\tilde{A}_{1}\langle$ say $\rangle$. Then $\operatorname{Int}\left(A_{1} \cup A_{2}\right)=\operatorname{Int} A_{2} \cup \operatorname{Int} \tilde{A}_{1}$ and Int $A_{2} \cap \operatorname{Int} \tilde{A}_{1}$ is open and non-null. Then if $B=\left(\right.$ Int $\left.A_{2}\right) \cup\left(\right.$ Int $\left.\tilde{A}_{1}\right), B$ is collared,4 and, in fact bi-collared. Hence $A_{1} \cup A_{2}$ is nice in $E^{n+1}$.

PROOF OF THE THEOREM. Let $p$ be a point of $Y$ and $\epsilon$ sufficiently small that $S(p, \epsilon) \cap(Y \backslash p)=\square$ (the empty set).

Let $B_{1}, B_{2}, \cdots$ be a sequence of balls with diameter approaching zero that are "concentric" about $p$, each of which meets $M$ nicely, as guaranteed by the condition $\left(E^{n}, \dot{B}_{i}^{n} \cap M \times I^{2}\right) \approx\left(E^{n}, S^{n-2} \times I^{2}\right)$. The spheres $\dot{B}_{1}, \dot{B}_{2}, \cdots$ maybe taken disjoint. Let $\dot{B}_{i}$ and $\dot{B}_{i+1}$ determine an annulus $A_{i}$ on $M$. Since $\dot{B}_{t} \cap M$ is nice in $M$, a homeomorphism of $B_{t}$ onto itself moving points an arbitrarily small amount may be defined to insure $A_{i}$ is an annulus. The boundary components of $A_{i}$ are denoted by $S_{i}$ and $S_{i+1}$. Let $B_{i}$ be decomposed by $S_{i}$ into two components $C_{i}^{N}$ and $C_{i}^{S}$, whose closures are closed $n-1$ disks and the notation is chosen so that $C_{1}^{N}, C_{2}^{N}, \cdots$ all lie on the same side of $E^{n} \backslash M$. Since $S^{n-2}$ is nicely embedded in $E^{n}$, it is clear that the consistency conditions required in the hypotheses of $3^{\prime}$ above hold for $S_{i}$ relative to $A_{i}$ and $C_{i}^{N}$. Hence $A_{i} \cup C_{i}^{N}$ is a nice disk $F_{i}$. Since $S_{i+1}$ is nice relative to $C_{i+1}^{N}$ and $A_{i}, C_{i+1}^{N} \cup A_{i}$ is a nice $n-1$ disk $G_{i}$. The conditions of 3 (above) are fulfilled so that $F_{i} \cup G_{i}$ is a flat $n-1$ sphere. By passing $n-1$ planes parallel to the base of an $n$-simplex that converge to the $a$

${ }^{3}$ Such a homeomorphism is easily found via the plane Schoenflies theorem.

- This is the content of Lemma 4 of [1]. 
vertex, one may slice the $n$-simplex into a sequence of nice $n$-cells $\sigma_{1}^{n}, \ldots$ with diameters approaching zero. By mapping each $\sigma_{i}^{n}$ to $F_{i} \cup G_{i}$ so that the consecutive functions agree on the common face of $\sigma_{i}^{n}$ and $\sigma_{i+1}^{n}$, the manifold $M$ is seen to have a collar at $p$ relative to the complementary domain determined by $C_{1}^{N}, \ldots$ A similar construction of the other side of $M$ shows that $M$ is in fact locally bi-collared at $p$.

By noting that the set $Y$ of $M$ consisting of points where $M$ fails to be locally flat is closed, it is easy to extend the above theorem to the case cardinal of $Y \leqq \mathcal{F} C_{0}$.

Added in proof. Corollary. If $S$ is an $\mathrm{n}-1$ sphere that is locally flat except possibly at two points $p$ and $q$ and if $S$ is $L W F$ at either $p$ or $q$, then $S$ is flat. 5

A question we have been unable to resolve is contained in the following.

PROBLEM. If $M^{n-1}$ is LWF, is it LF in $M^{n}$ ? The result is known to be true for $n=3$.

\section{REFERENCES}

1. M. Brown, Locally flat embeddings of topological manifolds, Ann. of Math. 75 (1962), 331-341.

2. J. C. Cantrell, Almost locally flat embeddings of $S^{n-1}$ in $S^{n}$, Bull. Amer. Math. Soc. 69 (1963), 716-718.

3. O. G. Harrold, Combinatorial structures, local unknottedness and local peripheral unknottedness, Topology of 3-manifolds and related topics, Prentice-Hall, Englewood Cliffs, N. J., 1962; pp. 71-83.

4. - Locally peripherally unknotted surfaces in $E^{3}$, Ann. of Math. 69 (1959), 276-290.

5. - Locally tame curves and surfaces in three-dimensional manifolds, Bull. Amer. Math. Soc. 63 (1957), 298.

Florida State University

5 One may define a concept of $M^{n-1}$ being LWF with respect to the complementary domain A (or the other complementary domain B) and derive a similar result. 